\title{
Spooks generated by brain-body mismatch
}

Illusory feeling that someone is behind you may be caused by faulty integration of signals from the body.

\section{Alison Abbott}

06 November 2014

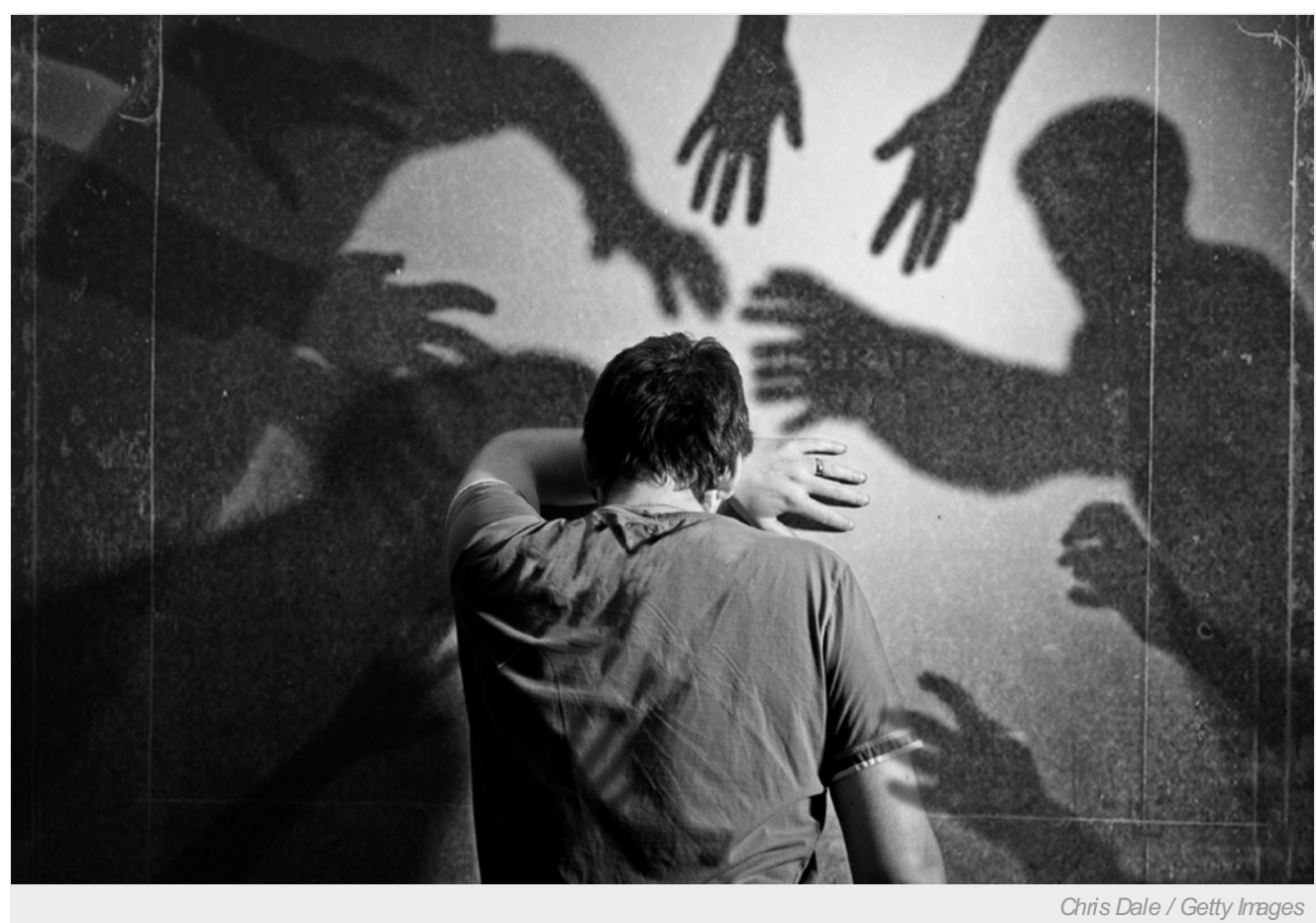

Apparitions have been a mainstay of fiction and drama for centuries, from Hamlet's father to Bathsheba in last year's smash hit horror movie The Conjuring. But under some circumstances, real people can also believe that a non-existent person is close to them extreme mountaineers often report the phenomenon, for example.

Some people with relatively rare types of brain injury also experience this 'feeling of a presence'. A study involving a dozen such patients, published today in Current Biology ${ }^{1}$, suggests that the eerie feeling may arise when a person's brain fails to integrate properly the different signals it receives from the limbs, such as those generated by touch and information about their position in space. The researchers also reproduced the illusion in healthy volunteers in the lab, with the help of purpose-built robots.

"This is the first systematic study of a phenomenon that is quite well known, but rarely looked at biologically," says Henrik Ehrsson, who studies self-perception at the Karolinska Institute in Stockholm. "It is fascinating."

In the study, the research team of neuroscientists and roboticists used multiple types of brain imaging to work out which regions of the brain were injured in patients with tendencies to experience feelings of a presence. They compared these regions with those injured in control patients who had experienced other types of complex hallucinations.

The only region uniquely associated with the feeling of a presence was the frontoparietal cortex, where different types of sensory and motor information are integrated. Two other regions, the temporoparietal cortex and the insula, were also damaged in both the study participants and the control patients. All three regions have been associated with bodily self-consciousness ${ }^{2}$.

The results show that not all types of spookiness emerge in the same way from the brain. "They show that the neural networks involved in the feeling of a presence are not the same as those involved in out-of-body experiences or in seeing a doppelgänger," says the lead author of the study, cognitive neuroscientist Olaf Blanke of the Swiss Federal Institute of Technology of Lausanne (EPFL).

Blanke suspected that the effect could be due to the brain misperceiving the source and identity of signals from the body generated by touch, perception of one's position in space (also known as proprioception) and movement. He designed experiments to see whether it 
was possible to induce the illusion in healthy volunteers, by confusing them about what was touching them.

\section{Slave to timing}

The team set up a master-slave robotic system to test the proposition. In a series of experiments, participants - who were unaware of the researchers' purposes - had to move a handle on the master robot in front of them with their right index finger; a slave robot behind them would then touch them on the back with a similar movement, either right away or with a half-second delay.

That touch was the only sensory information about their environment that the participants received: they were blindfolded and wore headphones delivering white noise to mask the sounds of the robots' movements.

When the robot's feedback was immediate, most participants soon began to feel that they were touching themselves, even though they were reaching forwards. But when there was a delay, they felt more often that the touch was coming from someone, or something, else. Many had a spooky feeling that a presence was close behind them doing the touching. They also felt that their bodies were farther back in the room than they really were, and closer to the invisible presence.

In a further experiment, another set of participants was put in front of four people who were chatting, and told that one or more of them may be in the same room when they carried out the test. During the test, the delayed touch led them to feel that there were several people in the room - even though they remained alone.

The illusions were caused by the mismatch between the expected and actual sensory information, says Blanke. He now wants to scan the brains of healthy volunteers while they do the test, to see whether the brain area that activates in them is the same as the one that is damaged in the patients.

Disrupting the brain's ability to recognize its own body teaches us something very fundamental about ourselves, says Giulio Rognini, one of the EPFL experimenters. When the brain malfunctions it can "sometimes create a second representation of the body, which is no longer perceived as 'me' but as someone else, a presence", he says.

The experiments may also shed light on the origin of some hallucinations experienced by people with schizophrenia, say the researchers.

Nature | doi:10.1038/nature.2014.16294

\section{References}

1. Blanke, O. et al. Curr. Biol. http://dx.doi.org/10.1016/j.cub.2014.09.049 (2014).

2. Blanke, O. et al. Nature Rev. Neurosci. 13, 556-571 (2012). 